\section{Protokoll der Mitgliederversammlung}

Aus Gründen der Lesbarkeit wird im Protokoll bei der Nennung der Teilnehmer auf die akademischen Titel sowie geschlechtsbezogene Formulierungen verzichtet.

Allgemeine Personenbezeichnungen gelten gleichwohl für jedwedes Geschlecht $(\mathrm{m} / \mathrm{w} / \mathrm{d})$.

Termin: Mittwoch, 23. September 2020

17.30-20.17 Uhr

Anwesend: 79 Mitglieder

Die Mitgliederversammlung wird auf Grundlage von Art. 2 GesRuaCOVBekG ${ }^{1}$ „im Wege der elektronischen Kommunikation“ durchgeführt. Die Übertragung der virtuellen Mitgliederversammlung erfolgt aus dem Versammlungsraum in den Räumlichkeiten der Geschäftsstelle der DGN.

Einleitend weist der DGN-Geschäftsführer Jonas auf technische Aspekte der ersten digital durchgeführten Mitgliederversammlung hin.

\section{TOP 1: Begrüßung und Totengedenken}

Der Präsident, Krause, eröffnet die Mitgliederversammlung und begrüßt die Anwesenden. Die Mitglieder gedenken der seit der letzten Mitgliederversammlung Verstorbenen.

TOP 2: Feststellung der ordnungsgemäßen Einladung und der Beschlussfähigkeit der Mitgliederversammlung

Die Einladung erfolgte am 30. Juli 2020 und somit gemäß § 18 III der DGN-Satzung. Die Mitgliederversammlung ist damit beschlussfähig.

1 Gesetz über Maßnahmen im Gesellschafts-, Genossenschafts-, Vereins-, Stiftungs- und Wohnungseigentumsrecht zur Bekämpfung der Auswirkungen der COVID-19-Pandemie
TOP 3: Genehmigung der Tagesordnung

Die Tagesordnung wird ohne Änderungen einstimmig angenommen.

TOP 4: Genehmigung des Protokolls der Mitgliederversammlung vom 5. April 2019 in Bremen

Das Protokoll der Mitgliederversammlung vom 5. April 2019 in Bremen wird ohne Gegenstimme angenommen.

\section{TOP 5: Bericht des Präsidenten}

Krause geht in seinem Bericht insbesondere auf folgende Punkte ein:

- finanzielle Situation der DGN

- Umstrukturierung der Geschäftsstelle

- Auswirkungen der COVID-19-Krise auf die DGN allgemein und die Jahrestagung im Besonderen

Bedeutende Themen für das 4. Quartal 2020:

- Weiterentwicklung der Geschäftsstelle durch eine hierfür initiierte Strukturkommission

- DGN-Jahrestagung 2021

- Haushalt 2021

Weitere relevante Themen 2020 und darüber hinaus:

- stationäre nuklearmedizinische Therapien

- Radiopharmazie (u. a. vor dem Hintergrund der Theranostik-Entwicklung)

- S3-Leitlinie „Schilddrüsenkarzinom“

- VISION-Studie - Umsetzung an deutschen Zentren

- G-BA-Erprobungsstudie zur Beta-Amyloid-PET/CT bei Demenz unklarer Ätiologie nach $\S 137$ e SGB V

- G-BA-Stellungnahmeverfahren zur Methode PET(/CT)

- PSMA-Verfügbarkeit in Deutschland Etablierung eines Compassionate Use Programms
TOP 6: Bericht des Kongresspräsidenten 2020

Essler berichtet über die 58. DGN-Jahrestagung: Diese fand infolge der COVID-19-Krise vom 7.-9. Juli 2020 erstmals online statt.

\section{Programm}

Für das wissenschaftliche Programm wurden in diesem Jahr 330 Abstracts eingereicht, von denen 311 angenommen wurden (Ablehnungsquote: 8,8\%).

Infolge der extrem kurzfristigen Umstellung des Veranstaltungsformats konnten im Rahmen der online durchgeführten Jahrestagung lediglich die Beiträge der 7 Leuchtturm- und 8 Fortbildungssitzungen als Vorträge des wissenschaftlichen Programms präsentiert werden. Auf diese Weise wurden die Highlights des Kongresses herausgestellt.

Das Angebot, die dadurch als Vortrag entfallenen Beiträge der übrigen wissenschaftlichen Sitzungen als ePoster einzureichen, wurde von der Mehrheit der Autoren angenommen.

Der Zugang zum Postkongress-Symposium „Innovation auf dem Prüfstand! SPECT/CT vs. PET/CT vs. PET/MRT - Was brauchen wir in Klinik und Praxis?" wurde im Zuge der Umstellung auf die digitale Jahrestagung allen Teilnehmern kostenfrei zur Verfügung gestellt.

Weitere besondere Veranstaltungen bzw. Beiträge im Rahmen der Jahrestagung waren:

- Eröffnungsveranstaltung mit WolfgangBecker-Gedächtnisvorlesung:

„Dosimetrie der Radionuklidtherapie Alte und Neue Tage“ (Sattler, Leipzig)

- aktuelle Stunde - COVID-19

- Medizinphysik-Curriculum 


\section{Teilnehmer}

Zur Jahrestagung haben sich 1107 Teilnehmer angemeldet. Aufgrund des digitalen Formats wurden erstmals Gruppen- sowie Institutsanmeldungen angeboten. Dabei wurden die Institutsanmeldungen mit je 15 Teilnehmern kalkuliert.

\section{Industriebeteiligung}

Auch die Industrieausstellung konnte digital umgesetzt werden. Daran beteiligten sich 32 von 57 Ausstellern (56\%) der ursprünglich geplanten Präsenzausstellung. Darüber hinaus fanden 9 Lunchsymposien online statt.

Der Kongresspräsident dankt allen beteiligten Referenten, den Teilnehmern, Industriepartnern, dem Vorstand und nmi-Geschäftsführer.

Der DGN-Präsident dankt dem Kongresspräsidenten für dessen Engagement.

\section{TOP 7: Bericht zur Jahrestagung 2021}

Der Geschäftsführer der nmi GmbH, Herrmann, berichtet, dass auch die NuklearMedizin 2021 angesichts des ungewissen Endes der COVID-19-Krise als virtuelle Jahrestagung durchgeführt werden soll. Diese soll allabendlich jedoch um eine an einem jeweils anderen Ort durchgeführte Präsenzveranstaltung ergänzt werden.

Diese Abendveranstaltungen sollen mit einer Leuchtturm-Sitzung beginnen, die für die virtuell anwesenden Teilnehmer live übertragen wird. Im Anschluss kann ein von der Industrie gesponsortes Get-Together stattfinden. Darüber hinaus wird der jeweiligen Regionalgesellschaft angeboten, sich als Kooperationspartner in die Abendveranstaltung einzubringen. Des Weiteren wird ein 3. Stream für wissenschaftliche Vorträge in das Programm NuklearMedizin 2021 aufgenommen werden.

\section{TOP 8: Bericht des Ausschusskoordinators}

Luster gibt einen Überblick über die Arbeit der Ausschüsse:

Die ausführlichen Berichte der Ausschüsse, Arbeitsgemeinschaften und Regionalgesell- schaften für den Berichtzeitraum sind in Ausgabe 2/2020 der Nuklearmedizin sowie über die Homepage des Thieme-Verlags veröffentlicht.

Das Angebot, die für 2020 geplanten Sitzungen digital durchzuführen, wurde von 10 der 12 Ausschüsse angenommen. Die Teilnahmequote an den einzelnen Sitzungen ist - mit jeweils mindestens $70 \%$ durchweg positiv.

Abschließend spricht Luster in einem persönlichen Ausblick Themen an, die seines Erachtens für die Zukunft der DGN besondere Relevanz haben werden:

- strukturelle Anpassung der Geschäftsstelle

- Austausch mit Politik und Behörden, anderen Fachgesellschaften und der Industrie

- Studien zur Evidenzgenerierung

- Nachwuchsgewinnung

\section{TOP 9: Bericht aus der Schriftleitung der Zeitschrift „Nuklearmedizin“}

Essler hat die Schriftleitung zum 1. Januar 2020 von Kotzerke übernommen.

Er geht in seinem Bericht u. a. auf folgende Punkte ein:

- Einreichung von Artikeln nach

- Rubrik sowie

- Ländern und deutschen Standorten

- Entwicklung des Impact Factors

- Änderungen bei den Sektionseditoren

Anmerkung: Die 3 nachfolgend genannten TOPs werden zusammen behandelt:

\section{TOP 10: Bericht des Kassenführers}

\section{TOP 11: Finanzanalyse DGN}

\section{TOP 12: DGN-Haushalt 2020}

Freudenberg berichtet zunächst vom vergangenen Geschäftsjahr; im Anschluss folgt eine weitergehende Analyse der Entwicklung der finanziellen Situation:

\section{Bericht des Kassenführers}

Die Einnahmen sind um 32 522,41€ im Vergleich zum Vorjahr gestiegen (leichte gestiegene Einnahmen durch Mitgliedsbei- träge, Einnahmen aus Veranstaltungen sowie Förderung der Erstellung der S3-Leitlinie „Schilddrüsenkarzinom“ durch die Deutsche Krebshilfe (AWMF-RegisterNr. 031-056OL) durchlaufender Posten).

Die Höhe der Ausgaben für die vom Vorstand beschlossenen Projekte, welche sämtlich der Erfüllung des Vereinszwecks dienen, ist im Vergleich zum Vorjahr um $3879,23 €$ gestiegen.

Die Gesamtausgaben sind um 22 197,45€ im Vergleich zum Vorjahr gestiegen.

Im Ergebnis sind der Einnahmeüberschuss der DGN auf 11823,41€ und damit das Vermögen auf $94830,77 €$ gestiegen.

Der scheinbare Anstieg des DGN-Vermögens ist jedoch in der Zusammenschau mit der Abschmelzung des nmi-Vermögens zu sehen. Dieses ist in den Jahren 2016-2019 insbesondere infolge der stark zurückgehenden Kongresseinnahmen um $377704 €$ gesunken.

Freudenberg weist darauf hin, dass die Entwicklung seit 2017 bekannt ist und Sparmaßnahmen beschlossen und umgesetzt wurden. Durch die COVID-19-Krise und insbesondere die damit einhergehende fehlende Planungssicherheit hat sich die Situation nochmals verschärft. In der Folge muss es weitere Änderungen in DGN und nmi geben.

TOP 13: DGN-Finanzen - eine strategische Standortbestimmung

Vor dem Hintergrund des zuvor vorgestellten Sachverhalts geht Krause auf folgende Punkte ein:

- Wie von Freudenberg dargestellt, ist die finanzielle Situation nicht ursächlich der COVID-19-Krise geschuldet.

- Es gilt, in allen Bereichen zu sparen. Dies schlägt sich bereits im DGN-Haushalt des laufenden Jahres nieder.

- DGN-Vorstand, nmi-Geschäftsführer und vokativ beschäftigen sich bereits seit geraumer Zeit mit Konzepten und Destinationen für die DGN-Jahrestagungen, um das Jahresergebnis der nmi GmbH zu optimieren. Darüber hinaus gab es seitens der nmi $\mathrm{GmbH}$ bereits Anpassungen der Teilnahmegebühren für Kongress, Festabend und Präsidentenessen. 
- Die Vorstandsmitglieder verzichten auf Reisekosten des laufenden Jahres.

- Es ist von herausragender Wichtigkeit, dass die Fachgesellschaft in der Lage bleibt Projekte durchzuführen, die für die Weiterentwicklung des Fachgebietes notwendig sind. Es muss daher Veränderungen und neue Strategien geben, um weiterhin solche Projekte durchführen zu können.

\section{Ausblick}

- Der Vorstand wird den Haushalt für das kommende Jahr in seiner nächsten Sitzung erörtern und beschließen. Gleichzeitig muss sichergestellt sein, dass Projekte für das Fachgebiet entwickelt und zukünftig auch durchgeführt werden (können).

- Die Umstrukturierung der Geschäftsstelle soll im Jahr 2021 zum Abschluss kommen.

- Auch die Mitglieder sind gefragt bzw. müssen sich fragen, wie sie sich einbringen können.

Abschließend bittet Krause die Mitglieder um ihre Unterstützung und ihr Vertrauen. Die DGN hat in den letzten Jahren große Entwicklungen vollzogen und wird dies auch weiterhin tun. Sie ist, eine wirtschaftlich solide Entwicklung vorausgesetzt, zukunftsfähig aufgestellt.

\section{TOP 14: Bericht aus der nmi GmbH}

Der Geschäftsführer der nmi GmbH, Herrmann, stellt den Jahresabschluss des Geschäftsjahres 2019 vor. Dieser weist einen Jahresüberschuss i. H.v. $89962,48 €$ aus.

Der Präsident dankt Herrmann für dessen Einsatz als Geschäftsführer der nmi GmbH.

\section{TOP 15: Bericht der Kassenprüfer}

Die Kasse wurde von den Kassenprüfern Kley und Ross geprüft. Diese berichten, vertreten durch Kley, dass die Kasse ordentlich und gut geführt ist und es keinen Anlass zur Beanstandung gibt.

\section{TOP 16: Entlastung des Vorstands}

Die Kassenprüfer, vertreten durch Kley, beantragen die Entlastung des Vorstands. Dieser Antrag wird mit 65 Ja-Stimmen bei 2 Gegenstimmen und 11 Enthaltungen angenommen.
TOP 17: Wahl der Kassenprüfer

Der Vorstand schlägt der Mitgliederversammlung Kley und Ross zur Wahl als Kassenprüfer für das Berichtsjahr 2020 vor.

Diese werden mit jeweils 75 Ja-Stimmen bei 3 Enthaltungen ohne Gegenstimmen gewählt. Beide erklären, dass sie die Wahl annehmen.

\section{TOP 18: Formale Aspekte der am 20. April 2018 beschlossenen Satzungsänderung}

Durch die am 20. April 2018 beschlossene Änderung der Satzung kommt es hinsichtlich der Einführung der Funktion des designierten Präsidenten als gewählten zukünftigen Präsidenten zu einer Regelungslücke.

Vor diesem Hintergrund fasst die Mitgliederversammlung mit 71 Ja-Stimmen bei 3 Gegenstimmen und einer Enthaltung folgenden Beschluss:

„Die Mitgliederversammlung hat am 20. April 2018 eine Änderung der Satzung beschlossen; diese wurde mit Eintragung in das Vereinsregister wirksam. Die nächste - und erste - turnusgemäße Wahl des Vorstands gemäß aktueller Satzung erfolgt voraussichtlich im Rahmen der Jahrestagung 2021. Der amtierende Vorstand wurde auf Grundlage der vor der letzten Satzungsänderung geltenden Bestimmungen gewählt, die die Funktion des designierten Präsidenten als gewählten zukünftigen Präsidenten nicht vorsah. Um die nächste Wahl des Vorstands vollumfänglich nach den Bestimmungen der geltenden Satzung gem. §9 II durchführen zu können und gleichzeitig die - mit der Satzungsänderung bezweckte - Einarbeitung des gewählten designierten Präsidenten zur Vorbereitung auf das zukünftige Amt zu gewährleisten, beschließt die Mitgliederversammlung, die Wahl des designierten Präsidenten als gewählten zukünftigen Präsidenten gem. § $8 \mathrm{lb}$. vorzuziehen und im Rahmen der am 23. September 2020 stattfindenden Mitgliederversammlung durchzuführen. Die Amtszeit des gewählten designierten Präsidenten als gewählter zukünftiger Präsident soll damit in Abweichung von §9 I ausnahmsweise bereits am Tage seiner Wahl beginnen. Er löst den (amtierenden) Präsidenten der Gesellschaft in seinem Amt als Präsident mit dem gem. §9 I geregelten Amts- antritt des Vorstands ab, der gemäß aktueller Satzung voraussichtlich im Rahmen der Jahrestagung 2021 gewählt wird.“

TOP 19: Wahl des designierten Präsidenten als gewählter zukünftiger Präsident (...) für die Amtsperiode 2021-2023

Der Vorstand hat der Mitgliederversammlung mit der Einladung - und damit formund fristgerecht gemäß §9 I DGN-Satzung - den amtierenden stellvertretenden Präsidenten, Luster, zur Wahl als designierter Präsident vorgeschlagen.

Weitere Wahlvorschläge wurden nicht eingereicht.

Luster wird mit 67 Ja-Stimmen bei 4 Gegenstimmen und 8 Enthaltungen gewählt. Er erklärt, dass er die Wahl annimmt.

\section{TOP 20: Satzungsänderung}

Krause erläutert, dass die Mitgliederversammlung nur ausnahmsweise auf Grundlage der vor dem Hintergrund der COVID19-Krise erlassenen Ausnahmeregelungen digital durchgeführt werden kann, obwohl dieses Versammlungsformat in der Satzung der DGN nicht ausdrücklich vorgesehen ist. Um eine digitale Durchführung von Mitgliederversammlungen wie auch Vorstandssitzungen zukünftig zu ermöglichen, schlägt der Vorstand folgende Beschlüsse bzw. Satzungsänderungen vor:

1. Satzungsänderung: Vorstandsversammlungen online oder in Schriftform (Ergänzung § $10 \mathrm{IV}$ )

Mit dem Vorschlag, das Format der Vorstandsversammlungen ausdrücklich auf die Durchführung von Online- oder kombinierten Präsenz-/Online-Versammlungen zu erweitern, soll einerseits die ordnungsgemäße und regelhafte Vereinsführung zu jeder Zeit sichergestellt sein - also auch z. B. während bestehender Gebote zu Kontaktbeschränkungen oder Erlasse von Dienstreiseverboten durch die Dienstherren der Amtsträger - und andererseits der ehrenamtliche Aufwand für die Amtsträger reduziert werden. Der Entwurf der vorgeschlagenen Satzungsänderung ist der Einladung als Anlage 1 beigefügt. 
Die Satzungsänderung wird mit 71 JaStimmen bei 3 Enthaltungen ohne Gegenstimmen beschlossen.

2. Mitgliederversammlung

(Ergänzung § 18 II und III)

Mit dem Vorschlag, Mitgliederversammlungen sowohl als Präsenzveranstaltung als auch online oder als kombinierte Präsenz-/Online-Veranstaltung durchführen zu können, soll die satzungsgemäß festgelegte jährliche Versammlungsmöglichkeit der Mitglieder garantiert werden. Dies betrifft insbesondere die für die Vereinsführung erforderlichen Beschlüsse der jährlich stattfindenden ordentlichen Mitgliederversammlungen sowie die ordnungsgemäße und regelhafte Würdigung der Amtsführung des Vorstands durch die Mitgliederversammlung.

Der Entwurf der vorgeschlagenen Satzungsänderung ist der Einladung als Anlage 1 beigefügt.

Die Satzungsänderung wird mit 71 JaStimmen bei 3 Enthaltungen ohne Gegenstimmen beschlossen.

\section{TOP 21: Stationäre nuklearmedizinische} Therapiezentren

Der Vorschlag für die Bearbeitung dieses Themas stammt aus dem Ausschuss Hochschulfragen. Im Anschluss wurden die Ausschüsse Radiopharmaka und Therapie vom Vorstand beauftragt, gemeinsam ein entsprechendes Konzept zu erarbeiten.

\section{Gründe für die Initiative}

Vonseiten der Selbstverwaltung wie auch der Gesundheitsministerien von Bund und Ländern werden Initiativen zur Einführung von Mindestmengen sowie Maßnahmen zur Sicherung der Strukturqualität erörtert. Das Land NRW (wie andere Bundesländer ebenfalls) hat dieses Thema bereits 2017 im Rahmen der Krankenhausplanung, bei der es ausschließlich um Therapiebetten geht, aufgegriffen, und diesbezüglich das Gespräch mit den Fachgesellschaften gesucht. Eine vorausschauende Positionierung der DGN ist daher angeraten.

Krause betont, dass es dabei ausschließlich um stationäre Therapien geht - ambulant durchgeführte Therapien sind nicht betroffen.
Neben einer Zahl zugelassener stationärer Therapien gibt es weitere, für die eine Zulassung zu erwarten ist (z. B. PSMA). Diese werden das nuklearmedizinische Versorgungsangebot zukünftig erweitern.

\section{Weiteres Vorgehen}

Im Nachgang des in den Ausschüssen und dem Präsidium diskutierten Entwurfs erfolgt nun die Erstellung eines Konzepts, das den Mitgliedern möglichst zur nächsten Mitgliederversammlung vorgestellt werden soll.

Es ist beabsichtigt, Berufsverband und Regionalgesellschaften in die Abfrage eines Meinungsbildes einzuschließen.

TOP 22: Aktuelle Entwicklungen in der Radiopharmazie

\section{Sachkenntnis nach § 15 AMG}

Das für Radiopharmazie zuständige Vorstandsmitglied Patt berichtet zu diesem Punkt:

Diese "Sachkenntnis“ ist in § 15 AMG geregelt. Voraussetzungen für sachkundige Personen sind demnach:

\section{- Studium der}

- Pharmazie,

- Chemie,

- pharmazeutischen Chemie und Technologie,

- Biologie,

- Humanmedizin oder

- Veterinärmedizin

- 3-jährige praktische Tätigkeit in der radiopharmazeutischen Chemie oder Nuklearmedizin

Dabei muss die praktische Tätigkeit nicht in einem Betrieb mit Herstellungserlaubnis nach §13 AMG absolviert werden, wie aus einem Rechtskommentar zum AMG hervorgeht (siehe Kügel, Müller, Hoffmann. Arzneimittelgesetz. 1. Auflage, 2011).

Ein Artikel zum genannten Thema wird in einer der nächsten Ausgaben der Nuklearmedizin veröffentlicht.

\section{Artikelreihe}

In Abstimmung mit dem Schriftleiter wird eine Reihe von Artikeln zu rechtlichen Aspekten der Radiopharmaka-Herstellung und -Anwendung im Fachjournal „Nuklearmedizin" erscheinen.

Hierfür sind zunächst ein Editorial sowie ein erster Artikel „Rechtliche Einordnung diagnostischer Radiopharmaka in der nuklearmedizinischen Forschung mit gesunden Probanden“ geplant.

Themenvorschläge oder zu behandelnde Fragen nimmt Patt gern entgegen.

\section{Treffen mit BMG}

Am 15. September 2020 fand ein Treffen mit dem BMG-Referat 114 „Arzneimittelentwicklung, -zulassung und -qualität, Großhandel, Klinische Prüfung“ statt.

Teilnehmer vonseiten der DGN waren:

- der stellvertretende Präsident, Luster,

- die Vorsitzenden des Ausschusses „Radiopharmaka“, Kuwert und Solbach, sowie

- Patt als weiteres Mitglied des Vorstands.

Erörterte Themen:

- Generatorverfügbarkeit

- Zulassungspflicht

- Radionuklidvorstufen

- Generatoren

- Kits

- mangelnde Definition Kits im AMG, nicht mehr zeitgemäße Definition in EU-Rechtsgebung

- Konsequenzen aus Zulassungspflicht

- AMRadV

- Erweiterung um Therapeutika

- Fallzahlerhöhung?

\section{PharmaSurvey EU}

Vonseiten der EU wurde eine öffentliche Konsultation zum Thema „Pharmazeutika: sichere und erschwingliche Arzneimittel“ durchgeführt. Diese diente der Identifikation insbesondere regulatorischer Probleme, die zu einer Einschränkung der Versorgung mit Pharmazeutika (und auch Radiopharmazeutika) führen können. 
TOP 23: Ort und Termin der nächsten Mitgliederversammlung

Die nächste Mitgliederversammlung wird vor dem Hintergrund der bestehenden Corona-Pandemie digital stattfinden. Der Termin ist noch bekannt zu geben.

\section{TOP 24: Verschiedenes}

Keine Punkte.

Der Versammlungsleiter schließt die Mitgliederversammlung um 20:17 Uhr.

gezeichnet

Prof. Dr. B. J. Krause

Präsident

Prof. Dr. P. Bartenstein

Schriftführer

Das Protokoll ist noch von der ordentlichen Mitgliederversammlung 2021 zu verabschieden.
VERANTWORTLICH FÜR DEN

INHALT

Deutsche Gesellschaft für

Nuklearmedizin e. V. (DGN)

Prof. Dr. Bernd Joachim Krause

(Präsident)

Nikolaistr. 29, 37073 Göttingen

E-Mail: office@nuklearmedizin.de

Schriftführer:

Prof. Dr. Peter Bartenstein

E-Mail: office@nuklearmedizin.de 